\title{
Estimación auscultatoria de la presión sistólica de arteria pulmonar: ¿Es factible?. Correlación con determinación ecocardiográfica
}

\author{
PAUL MCNAB, PABLO CASTRO, HUGO VERDEJO, GONZALO MARTÍNEZ
}

\section{Heart auscultatory assessment of pulmonary artery systolic pressure}

Background: The clinical assessment of pulmonary artery systolic pressure (PASP) by means of heart auscultation may be comparable to the measures obtained by Doppler echocardiography. Aim: To compare PASP estimated by heart auscultation and echocardiography. Material and Methods: Thirty consecutive patients aged $70 \pm 16$ years (68\% women) were evaluated prospectively, all of whom had an echocardiogram requested by their attending physician. Echocardiographic evaluation of PASP was made by tricuspid regurgitation velocity plus right atrial pressure estimate. Two trained clinicians, blinded for the clinical history and rest of physical examination, auscultated the patients. PASP was estimated from the auscultatory intensity of the pulmonary component in comparison to the aortic component of the second heart sound and its propagation towards the apex, in accordance to a pre-established algorithm. Correlation between auscultatory and echocardiographic measures of PASP was made by Pearson test. Variability between both methods and among observers was evaluated with Bland-Altman analysis. Results: Fifty two per cent of patients were hypertensive and 20\% diabetic. Admission diagnoses were heart failure in $50 \%$ of cases, exacerbation of chronic obstructive pulmonary disease in 20\%, pulmonary thromboembolism in 10\% and other clinical entities in 20\%. A significant correlation was found between auscultatory and echocardiographic estimation of PASP $(r=0.64, p=0,01)$. Bland-Altman analysis showed a mean difference between both determinations of $7.6 \pm 7.6 \mathrm{mmHg}$. Bland-Altman analysis between both operators showed an average difference of $5.4 \pm 8.4 \mathrm{~mm} \mathrm{Hg}$. Conclusions: Heart auscultatory evaluation allows a precise and reproducible estimation of PASP and compares favorably with echocardiographic assessment.

(Rev Med Chile 2010; 138: 1351-1356).

Key words: Echocardiography, Doppler; Heart auscultation; Pulmonary artery.

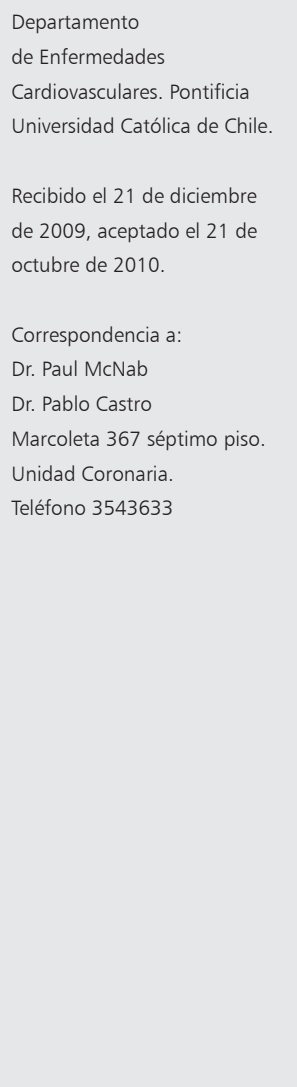

L a presión de arteria pulmonar es un parámetro de gran utilidad diagnóstica, pronóstica y terapéutica en una serie de patologías que se acompañan de hipertensión arterial pulmonar ${ }^{1}$. En pacientes con hipertensión pulmonar primaria la magnitud de la elevación de la presión sistólica de arteria pulmonar (PSAP), especialmente en ausencia de reversibilidad, se asocia a un pronós- tico desfavorable ${ }^{2}$. En pacientes con insuficiencia cardíaca crónica tanto isquémica como idiopática también constituye un predictor independiente de mortalidad $^{3,17}$. Su determinación en estos pacientes contribuye a evaluar el estado de la volemia y su elevación por sobre los valores basales precede a otros signos o síntomas de descompensación como la disnea y la aparición de edema. 
El valor de PSAP puede ser obtenido de forma invasiva, siendo lo más habitual en la práctica clínica el uso del catéter de Swan-Ganz, técnica descrita hace 38 años ${ }^{5}$. Si bien permite medir diversos parámetros hemodinámicos, su utilidad ha sido cuestionada por diversos autores ${ }^{6}$, en parte por los riesgos inherentes a su instalación ${ }^{7}$. La ecocardiografía Doppler revolucionó la evaluación de la PSAP al permitir su estimación de forma no invasiva mediante la velocidad del reflujo tricuspídeo y el cálculo en forma semi-cuantitativa de la presión de la aurícula derecha. Este método logra correlaciones con las mediciones con catéter entre 0,89 y $0,97^{8}$, con una variabilidad interobservador de $3 \%$. Su estimación se logra en la mayoría de los casos cuando la PSAP es mayor a $50 \mathrm{mmHg}$, en cambio cuando la PSAP está cercana a los valores normales la posibilidad de lograr medirla es de $72 \%{ }^{10}$. Otra técnica no invasiva, menos conocida, es a través del análisis espectral de los ruidos cardíacos. En ésta se realizan grabaciones digitales de los ruidos cardíacos, los cuales son convertidos e inscritos en curvas analógicas que representan cada ruido registrado, las que permiten estimar la PSAP por medio del análisis espectral del componente pulmonar del segundo ruido (P2), logrando una adecuada correlación con la medición ecocardiográfica e invasiva $(\mathrm{r}=0,84, \mathrm{DS} \pm 5,6 \mathrm{~mm} \mathrm{Hg}$, $\mathrm{p}<0,0001)^{11-13}$.

Del análisis de estas publicaciones y de la experiencia clínica es conocido que la intensidad de P2 se relaciona con la magnitud de la PSAP, así como también que la intensidad de éste va disminuyendo al alejarse del foco pulmonar, no así el componente aórtico del segundo ruido. De esta manera, creemos que es posible estimar la PSAP de forma clínica, mediante el método auscultatorio. Es por esto, y dado que no existen publicaciones previas que evalúen esta alternativa semiológica, nos pareció de interés investigar la correlación de este método con una técnica conocida y validada como la ecocardiografía Doppler.

\section{Método}

Se incluyeron 30 pacientes hospitalizados en nuestra institución, a quienes se les solicitó la realización de un ecocardiograma de superficie por su médico tratante y que presentaran una señal tricuspídea al Doppler confiable a criterio del ecocardiografista. Se excluyó a aquellos pacientes portadores de estenosis mitral con áreas menores a $1,5 \mathrm{~cm}^{2}$, prótesis valvular aórtica y presión arterial sistólica menor de 100 o mayor de $160 \mathrm{mmHg}$ al momento de la evaluación tanto ecocardiográfica como auscultatoria.

La estimación ecocardiográfica de la PSAP se realizó con un equipo GE Healthcare VIVID 7 Dimension. Se midió el jet regurgitante por imagen de Doppler contínuo en múltiples visiones, utilizándose aquella con peak de velocidad más alto para calcular la gradiente máxima durante el sístole, mediante la fórmula de Bernoulli modificada. La PSAP fue estimada como la gradiente de la válvula tricuspídea más la estimación de la presión de aurícula derecha (PAD), a través de la evaluación de los cambios del diámetro de la vena cava inferior (VCI) con la respiración no forzada:

$$
\mathrm{PSAP}=4 \times \mathrm{V}^{2}+\mathrm{PAD}
$$

Donde $\mathrm{V}=$ velocidad reflujo tricuspídeo.

$\mathrm{PAD}=5 \mathrm{mmHg}$ si la VCI es $<20 \mathrm{~mm}$ y colapsa $>50 \%$ en inspiración; $10 \mathrm{mmHg}$ si VCI es $<20$ mm y colapsa $<50 \%$ en inspiración; $15 \mathrm{mmHg}$ si VCI es $>20 \mathrm{~mm}$ y colapsa $>50 \%$ en inspiración; $20 \mathrm{mmHg}$ si VCI es $>20 \mathrm{~mm}$ y colapsa $<50 \%$ en inspiración ${ }^{14}$.

La auscultación fue realizada por separado por dos operadores con experiencia en esta técnica semiológica, en un plazo menor a 2 horas de haberse realizado el ecocardiograma, ciegos a la ficha, a la historia clínica y al resto del examen físico. Ambos utilizaron un estetoscopio 3M Littman ${ }^{\circledR}$ Master Classic II. El operador estimó la PSAP basándose en el espacio intercostal en que se igualaban las intensidades de ambos tonos del segundo ruido, según se muestra en la Figura 1.

El análisis estadístico se realizó buscando el grado de correlación entre la PSAP determinada por auscultación y la determinación por ecocardiografía mediante método de Pearson. La variabilidad entre los métodos y la variabilidad interobservador fue evaluada mediante análisis de Bland-Altman. Los resultados son expresados como promedio \pm desviación standard. La significancia estadística fue definida con un $p<0,05$.

\section{Resultados}

La edad promedio de los pacientes estudiados fue $70 \pm 16$ años (68\% mujeres). Los diagnósticos de ingreso fueron insuficiencia cardíaca descom- 
pensada en $50 \%$, enfermedad pulmonar obstructiva crónica reagudizada en $20 \%$, tromboembolismo pulmonar en $10 \%$ y otras patologías en el 20\% restante. El 53\% de los pacientes tenía antecedentes de hipertensión arterial, el 20\% de diabetes mellitus y un paciente presentó bloqueo completo de rama izquierda. El estudio ecocardiográfico mostró una fracción de eyección promedio de $59 \% \pm 11$ y presencia de insuficiencia mitral y tricuspídea moderada o severa en $12,3 \%$ y $33,3 \%$ respectivamente.

La PSAP promedio medida por auscultación fue de $42 \pm 12 \mathrm{mmHg}$ y por ecocardiografía fue de $41 \pm 12 \mathrm{mmHg}$, sin existir diferencia estadísticamente significativa entre ellas. Existió una correlación significativa entre la estimación auscultatoria y por ecocardiograma $(r=0,64, p=0,01)$. Figura 2. El análisis de Bland-Altman mostró una diferencia promedio entre ambas determinaciones de 7,63 \pm 7,6 mmHg. Figura 3 . Al analizar los pacientes con PSAP en el cuartil superior la diferencia fue menor $(1,2 \pm 11,8$ $\mathrm{mmHg}$ ), mientras que en el cuartil de PSAP más bajo la diferencia fue similar al análisis global $(7,7 \pm 20,6 \mathrm{mmHg})$. El análisis de Bland-Altman entre ambos operadores mostró una diferencia promedio entre las determinaciones de 5,4 $\pm 8,4 \mathrm{mmHg}$. Al revisar la distribución de la PSAP en nuestra población observamos que están representados valores desde los 25 hasta los 65 mmHg. Figura 4.

\section{Discusión}

En nuestro estudio la auscultación cardíaca por médicos entrenados con evaluación de la intensidad del componente pulmonar del segundo ruido respecto del componente aórtico y su grado de propagación al ápex del ventrículo izquierdo permitió estimar en forma precisa la PSAP al compararla con la ecocardiografía Doppler. Más aun, este método clínico tuvo una adecuada reproducibilidad al ser aplicado por dos examinadores distintos. Estos resultados están en consonancia con estudios previos que han logrado aproximarse con exactitud a la PSAP por medios fonocardiográficos ${ }^{11,16,24}$.

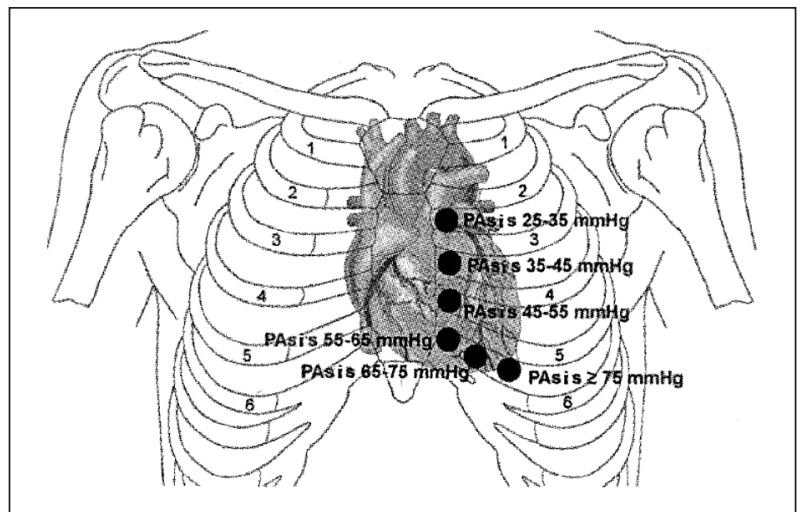

Figura 1. Algoritmo para la estimación de presión sistólica de arteria pulmonar por método auscultatorio. Modificado de Adams et al ${ }^{28}$. PAsis: Presión sistólica de arteria pulmonar.

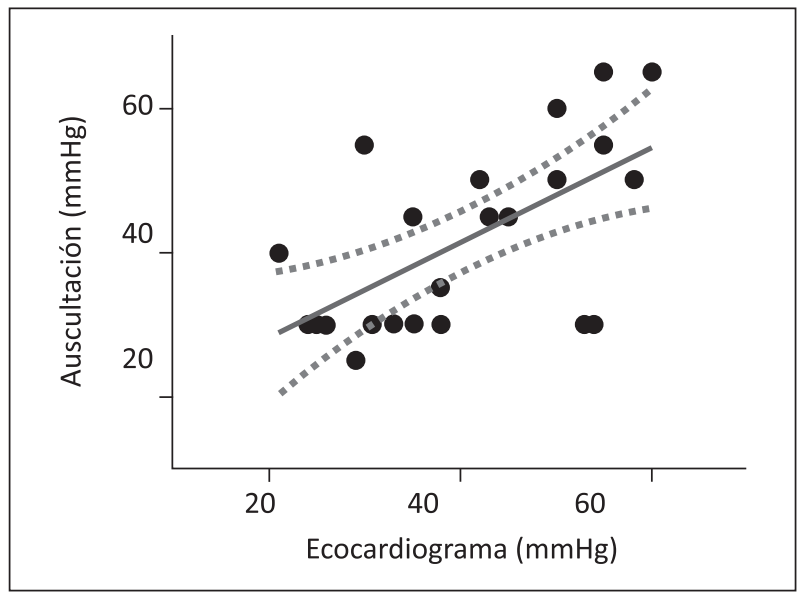

Figura 2. Correlación entre la estimación de PSAP mediante ecocardiografía y auscultación. PSAP: Presión sistólica de arteria pulmonar.

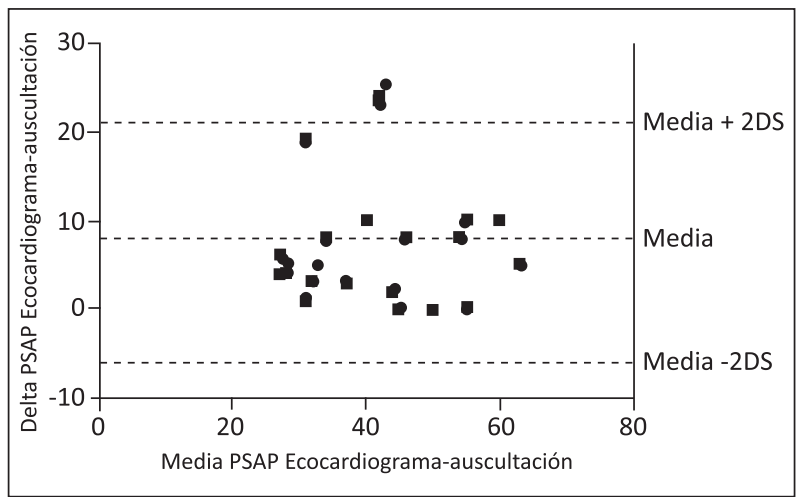

Figura 3. Variabilidad en la estimación de PSAP entre auscultación y ecocardiografía. PSAP: Presión sistólica de arteria pulmonar. 


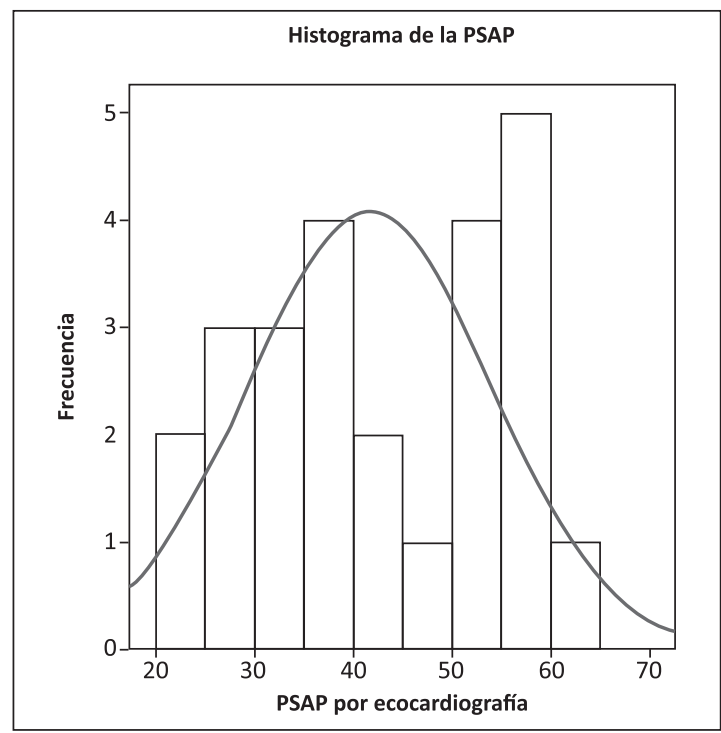

Figura 4. Distribución de PSAP medida por ecocardiografía en la muestra estudiada. PSAP: Presión sistólica de arteria pulmonar.

El segundo ruido cardíaco marca el fin de la sístole ventricular. Tiene dos componentes de alta frecuencia, que se relacionan con el cierre de las válvulas aórtica y pulmonar. Es conocido tanto del punto de vista clínico como mediante estudios fonocardiográficos que la intensidad del componente aórtico del segundo ruido se modifica según la presión arterial sistémica, aumentando en casos de hipertensión arterial y disminuyendo cuando la tensión arterial es baja. Esto se debe a que los cambios en la presión arterial modifican las presiones de distensión de las paredes arteriales, alterando la frecuencia resonante de la columna sanguínea ${ }^{26}$. Estas observaciones implican dos puntos prácticos. Primero, estos mismos fenómenos son aplicables a la circulación pulmonar y explican los cambios en P2 que ocurren con la hipertensión arterial pulmonar, lo cual ha sido corroborado con múltiples modelos matemáticos ${ }^{27}$. Segundo, las mediciones de PSAP estimadas mediante la comparación de la intensidad de los componentes del segundo ruido cardíaco se basan en una intensidad normal del componente aórtico. Es por esto que aquellos pacientes con presión arterial sistólica menor de 100 o mayor de $160 \mathrm{mmHg}$ fueron excluidos de nuestro estudio.

Conforme la medicina moderna se ha desarrollado de la mano de nuevas técnicas de laborato- rio, el arte de y la confianza en el examen clínico han disminuido progresivamente. Un examen cardiológico adecuado puede revelar una serie de hallazgos de gran importancia clínica, como la auscultación de soplos, un tercer ruido y cambios en la intensidad de los ruidos cardíacos norma$l^{1} s^{15}$. En este estudio se observó que la adecuada auscultación del segundo ruido pulmonar permite estimar la PSAP en una serie de condiciones clínicas frecuentes. De esta manera, se puede mejorar la impresión clínica al lado de la cama del paciente y potencialmente disminuir el uso de tecnologías más avanzadas, con los costos y riesgos que éstas pueden acarrear.

Es clara la importancia clínica y pronóstica que representa conocer la PSAP, tanto a corto como largo plazo, en variadas patologías como insuficiencia cardíaca aguda y crónica, tromboembolismo pulmonar, postoperatorio de cirugía cardíaca, enfermedades autoinmunes, etc ${ }^{17-23}$. Contar con un método clínico para estimar esta variable puede ser de gran utilidad en la evaluación periódica de estos pacientes, ayudando a la toma de decisiones terapéuticas en la práctica clínica diaria. Esto puede ser de aun mayor importancia en aquellos pacientes hospitalizados por descompensaciones de sus patologías crónicas, donde las mediciones pueden modificarse frecuentemente según la evolución del paciente.

El uso de este método clínico puede ser un complemento a otras técnicas ya descritas, como la ecocardiografía Doppler. Aun más, en condiciones en las cuales las señales de reflujo tricuspídeo al Doppler no son confiables o cuando no se logra o no se desea instalar un catéter de arteria pulmonar, el método auscultatorio puede ser de utilidad para no tener que obviar esta importante variable en las decisiones clínicas.

El número limitado de pacientes evaluados en este estudio puede dificultar su aplicación a grandes poblaciones de pacientes, afectando su reproducibilidad. Por otra parte, la utilización de la ecocardiografía Doppler como patrón de comparación puede ser discutible, toda vez que no corresponde al patrón de oro y sus mediciones están expuestas a un grado de variabilidad. Sin embargo, corresponde al método más utilizado en la práctica clínica para medir la PSAP y es sobre el cual se toman las decisiones clínicas habitualmente, de modo que tener una técnica clínica comparable con la ecocardiografía Doppler pa- 
rece ser adecuado. Además, publicaciones previas han mostrado una correlación apropiada de los métodos fonocardiográficos con las mediciones realizadas mediante un catéter de arteria pulmonar ${ }^{24,25}$. Finalmente, la técnica auscultatoria fue llevada a cabo por dos médicos cardiólogos con amplia experiencia y conocimiento del examen cardíaco, por lo que se desconoce la confiabilidad del examen realizado por individuos con menos experiencia. Un buen entrenamiento clínico puede subsanar esta dificultad, permitiendo obtener información confiable de una amplia gama de examinadores. Del mismo modo, expandir esta experiencia a médicos en formación entrenados en la medición auscultatoria de PSAP parece ser una alternativa razonable para continuar el estudio de esta técnica.

Por lo anterior, creemos que esta herramienta semiológica debiera ser incorporada a la práctica clínica habitual y enseñada junto con el resto del examen cardíaco, dado la gran utilidad de la información que aporta en una amplia variedad de patologías. Esperamos que la presente publicación ayude a promover y divulgar ésta técnica.

\section{Referencias}

1. Simonneau G, Galiè N, Rubin LJ, Langleben D, Seeger W, Domenighetti G, et al. Clinical classification of pulmonary hypertension. J Am Coll Cardiol 2004; 43: 5S-12S.

2. Okada O, Tanabe N, Yasuda J, Yoshida Y, Katoh K, Yamamoto T, et al. Prediction of life expectancy in patients with primary pulmonary hypertension. A retrospective nationwide survey from 1980-1990. Intern Med 1999; 38 (1): 12-6.

3. Komajda M, Jais JP, Reeves F, Goldfarb B, Bouhour JB, Juillieres $\mathrm{Y}$, et al. Factors predicting mortality in idiopathic dilated cardiomyopathy. Eur Heart J 1990; 11 (9): 824-31.

4. Verdejo HE, Castro PF, Concepción R, Ferrada MA, Alfaro MA, Alcaíno ME, et al. Comparison of a radiofrequency-based wireless pressure sensor to swan-ganz catheter and echocardiography for ambulatory assessment of pulmonary artery pressure in heart failure. J Am Coll Cardiol 2007; 50 (25): 2375-82.

5. Swan HJ. The pulmonary artery catheter in anesthesia practice. 1970. Anesthesiology 2005; 103 (4): 890-3.

6. Shah MR, Hasselblad V, Stevenson LW, Binanay C, O'Connor CM, Sopko G, et al. Impact of the pulmonary artery catheter in critically ill patients: meta-analysis of randomized clinical trials. JAMA 2005; 294 (13): 1664 70.

7. Binanay C, Califf RM, Hasselblad V, O'Connor CM, Shah MR, Sopko G, et al; ESCAPE Investigators and ESCAPE Study Coordinators. Evaluation study of congestive heart failure and pulmonary artery catheterization effectiveness: the ESCAPE trial. JAMA 2005; 294 (13): 1625-33.

8. Currie PJ, Seward JB, Chan KL, Fyfe DA, Hagler DJ, Mair $\mathrm{DD}$, et al. Continuous wave Doppler determination of right ventricular pressure: a simultaneous Dopplercatheterization study in 127 patients. J Am Coll Cardiol 1985; 6 (4): 750-6.

9. Berger M, Haimowitz A, Van Tosh A, Berdoff RL, Goldberg E. Quantitative assessment of pulmonary hypertension in patients with tricuspid regurgitation using continuous wave Doppler ultrasound. J Am Coll Cardiol 1985; 6 (2): 359-65.

10. Chan KL, Currie PJ, Seward JB, Hagler DJ, Mair DD, Tajik AJ. Comparison of three Doppler ultrasound methods in the prediction of pulmonary artery pressure. J Am Coll Cardiol 1987; 9 (3): 549-54.

11. Chen D, Pibarot P, Honos G, Durand LG. Estimation of pulmonary artery pressure by spectral analysis of the second heart sound. Am J Cardiol 1996; 78: 785-9.

12. Sutton G, Harris A. Leatham Second heart sound in pulmonary hypertension. Br Heart J 1968; 30 (6): 743-5.

13. Aggio S, Baracca E, Longhini C, Brunazzi C, Longhini L, Musacci G, Fersini C. Noninvasive estimation of the pulmonary systolic pressure from the spectral analysis of the second heart sound. Acta Cardiol 1990; 45 (3): 199-202.

14. Sciomer S, Magrì D, Badagliacca R. Non-invasive assessment of pulmonary hypertension: Dopplerechocardiography. Pulm Pharmacol Ther 2007; 20 (2): 135-40.

15. Phoon CK. Estimation of pressure gradients by auscultation: an innovative and accurate physical examination technique. Am Heart J 2001; 141 (3): 500-6.

16. Tranulis C, Durand LG, Senhadji L, Pibarot P. Estimation of pulmonary arterial pressure by a neural network analysis using features based on time-frequency representations of the second heart sound. Med Biol Eng Comput 2002; 40 (2): 205-12.

17. Grigioni F, Potena L, Galiè N, Fallani F, Bigliardi M, Coccolo F, et al. Prognostic implications of serial assessments of pulmonary hypertension in severe chronic heart failure. J Heart Lung Transplant. 2006; 25 (10): 1241-6.

18. Lai HC, Lai HC, Wang KY, Lee WL, Ting CT, Liu TJ. Severe pulmonary hypertension complicates postoperative 
outcome of non-cardiac surgery. Br J Anaesth 2007; 99 (2): 184-90.

19. Ramakrishna G, Sprung J, Ravi BS, Chandrasekaran K, McGoon MD. Impact of pulmonary hypertension on the outcomes of noncardiac surgery: predictors of perioperative morbidity and mortality. J Am Coll Cardiol 2005; 45 (10): 1691-9.

20. Villar J, Blazquez MA, Lubillo S, Quintana J, Manzano JL. Pulmonary hypertension in acute respiratory failure. Crit Care Med 1989; 17 (6): 523-6.

21. Carneiro AC, Barbosa IP, Chaves FC. CREST syndrome and pulmonary hypertension: a dark prognosis. Acta Med Port 2004; 17 (5): 409-14.

22. Toosi MS, Merlino JD, Leeper KV. Prognostic value of the shock index along with transthoracic echocardiography in risk stratification of patients with acute pulmonary embolism. Am J Cardiol 2008; 101 (5): 700-5.

23. Cappola TP, Felker GM, Kao WH, Hare JM, Baughman KL, Kasper EK. Pulmonary hypertension and risk of death in cardiomyopathy: patients with myocarditis are at higher risk. Circulation 2002; 105 (14): 1663-8.
24. Xu J, Durand LG, Pibarot P. A new, simple and accurate method for non-invasive estimation of pulmonary artery pressure. Heart 2002; 88 (1): 76-80.

25. Popov B, Sierra G, Durand LG, Xu J, Pibarot P, Agarwal $\mathrm{R}$, et al. Automated extraction of aortic and pulmonary components of the second heart sound for the estimation of pulmonary artery pressure. Conf Proc IEEE Eng Med Biol Soc 2004; 2: 921-4.

26. Zhang XY, Zhang YT. Model-based analysis of effects of systolic blood pressure on frequency characteristics of the second heart sound. Conf Proc IEEE Eng Med Biol Soc 2006; 6: 2888-91.

27. Reyes BA, Charleston-Villalobos S, González-Camarena $\mathrm{R}$, Aljama-Corrales T. Time-frequency representations for second heart sound analysis. Conf Proc IEEE Eng Med Biol Soc 2008; 8: 3616-9.

28. Adams KF Jr MD, Armstrong PW MD, Baughman KL MD, Binkley PF MD, Bourge RC MD, Bristow MR MD, et al. Nuggets, Pearls, and Vignettes of Master Heart Failure Clinicians. Congestive Heart Failure Volume 2001; 7:245-9. 